\title{
The VSOP Survey: Final individual results
}

\author{
R. Dodson ${ }^{*} a \dagger$ S. Horiuchi ${ }^{b}$, W. Scott ${ }^{c}$, E. Fomalont ${ }^{d}$, Z. Paragi ${ }^{e}$, S. Frey ${ }^{f}$, K. Wiik ${ }^{g}, \mathbf{H}$. \\ Hirabayashi $^{a}$, P. Edwards ${ }^{h}$, Y. Murata ${ }^{a}$, G. Moellenbrock ${ }^{d}$, L. Gurvits ${ }^{e}$, and S. Tingay ${ }^{b}$ \\ ${ }^{a}$ ISAS, JAXA, 3-1-1 Yoshinodai, Sagamihara, Japan \\ ${ }^{b}$ Centre for Astrophysics and Supercomputing, University of Swinburne, Melbourne, Australia \\ ${ }^{c}$ Physics and Astronomy Department, University of Calgary, Canada \\ ${ }^{d}$ National Radio Astronomy Observatory, Charlottesville, U.S. \\ e Joint Institute for VLBI in Europe, Dwingeloo, Netherlands \\ ${ }^{f}$ FÖMI Satellite Geodetic Observatory, Budapest, Hungary \\ ${ }^{g}$ Tuorla Observatory, Piikkiö, Finland \\ ${ }^{h}$ Australia Telescope National Facility, CSIRO, Sydney, Australia \\ E-mail: rdodson@vsop.isas.jaxa.jp
}

In February 1997 the Japanese radio astronomy satellite HALCA was launched to provide the space-borne element for the VSOP mission. HALCA provided linear baselines three-times greater than that of ground arrays, thus providing higher resolution and higher AGN brightness temperature measurements and limits. Twenty-five percent of the scientific time of the mission was devoted to the "VSOP survey" of bright, compact, extra-galactic radio sources at $5 \mathrm{GHz}$. A complete list of 294 survey targets were selected from pre-launch surveys, $91 \%$ of which were observed during the satellite's lifetime. The major goals of the VSOP Survey are statistical in nature: to determine the brightness temperature and approximate structure, to provide a source list for use with future space VLBI missions, and to compare radio properties with other data throughout the electromagnetic spectrum. All the data collected have now been analysed and is being prepared for the final image Survey paper. In this paper we present details of the mission, images of the sources, and some statistics of the contributions and completeness.

The 8th European VLBI Network Symposium

September 26-29, 2006

Toruń, Poland

\footnotetext{
*Speaker.

†Present address: Marie Curie Fellow, OAN, Alcalá de Heneres, España
} 

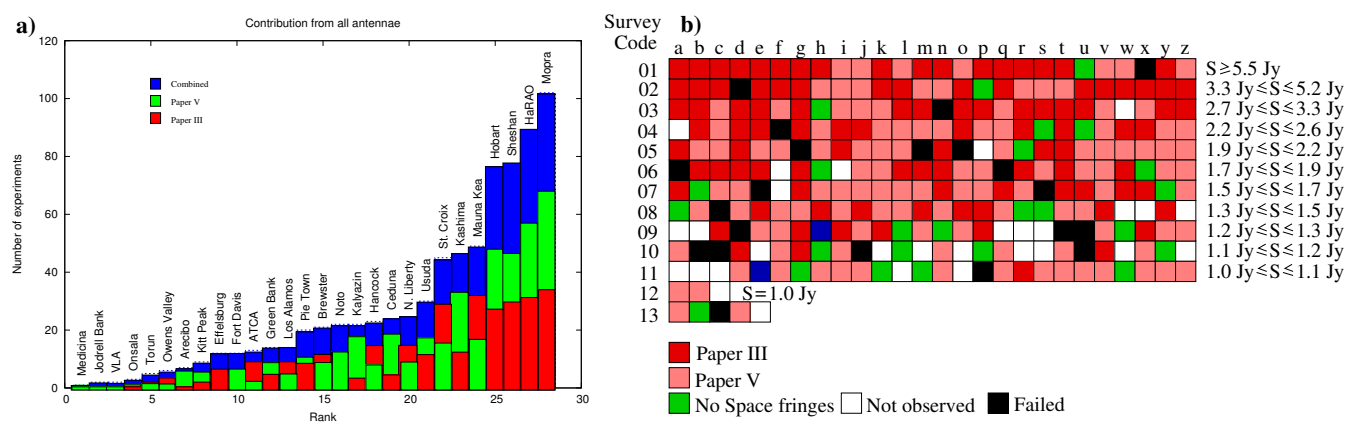

Figure 1: a) A histogram of the contribution of all 28 antennae used to make the VSOP survey images, for Paper III, V and both. b) The final status of all the VSOP survey targets.

\section{Introduction}

The VLBI Space Observing Program (VSOP) satellite HALCA provided the space baseline for the observation of a complete sample of bright compact Active Galactic Nuclei (AGNs) at $5 \mathrm{GHz}$; the VSOP survey [2]. Of this set of 294 AGNs: 102 were presented in [4] (hereafter P-III), 140 will be presented in [1] (hereafter P-V), and 29 were not observed. The remaining 23 did not produce space fringes, where we expected to find them. These maybe the true result, however we have erred on the side of caution and consider them to be failures. Figure 1a) plots the contribution of all the antennae to the published, or to be published, experiments, and Figure 1b) plots the final status of all the survey experiments.

The images produced are compared, where possible, to both the General Observing Time (GOT), and any other available, images. We confirmed, as reported in [3], that the Survey data reproduces that of the more completely sampled images. See Figure 2 which plots all the images from P-V.

\section{Conclusions}

We have finished the VSOP survey imaging data reduction. The paper covering 140 sources is in preparation, and when it is published the imaging portion of the VSOP Survey Project will be completed. All the images from the final paper are shown in a montage.

\section{Acknowledgments}

The contributions of very many people have been essential to the success of the VSOP Survey project. Many more than can be included in the list of authors. We wish to recognise and thank them for their contributions.

\section{References}

[1] Dodson, R., et al., 2007, in preparation, $\mathrm{P}-\mathrm{V}$

[2] Hirabayashi H., et al., 2000, PASJ, 52, 997 , P-I

[3] Lister M. L., Piner B. G., Tingay S. J., 2000, Astrophysical Phenomena Revealed by SVLBI, Proceedings of the VSOP Symposium, eds: H. Hirabayashi, P. G. Edwards, \& D. W. Murphy, p. 189

[4] Scott W. K., et al., 2004, ApJS, 155, 33, P-III 


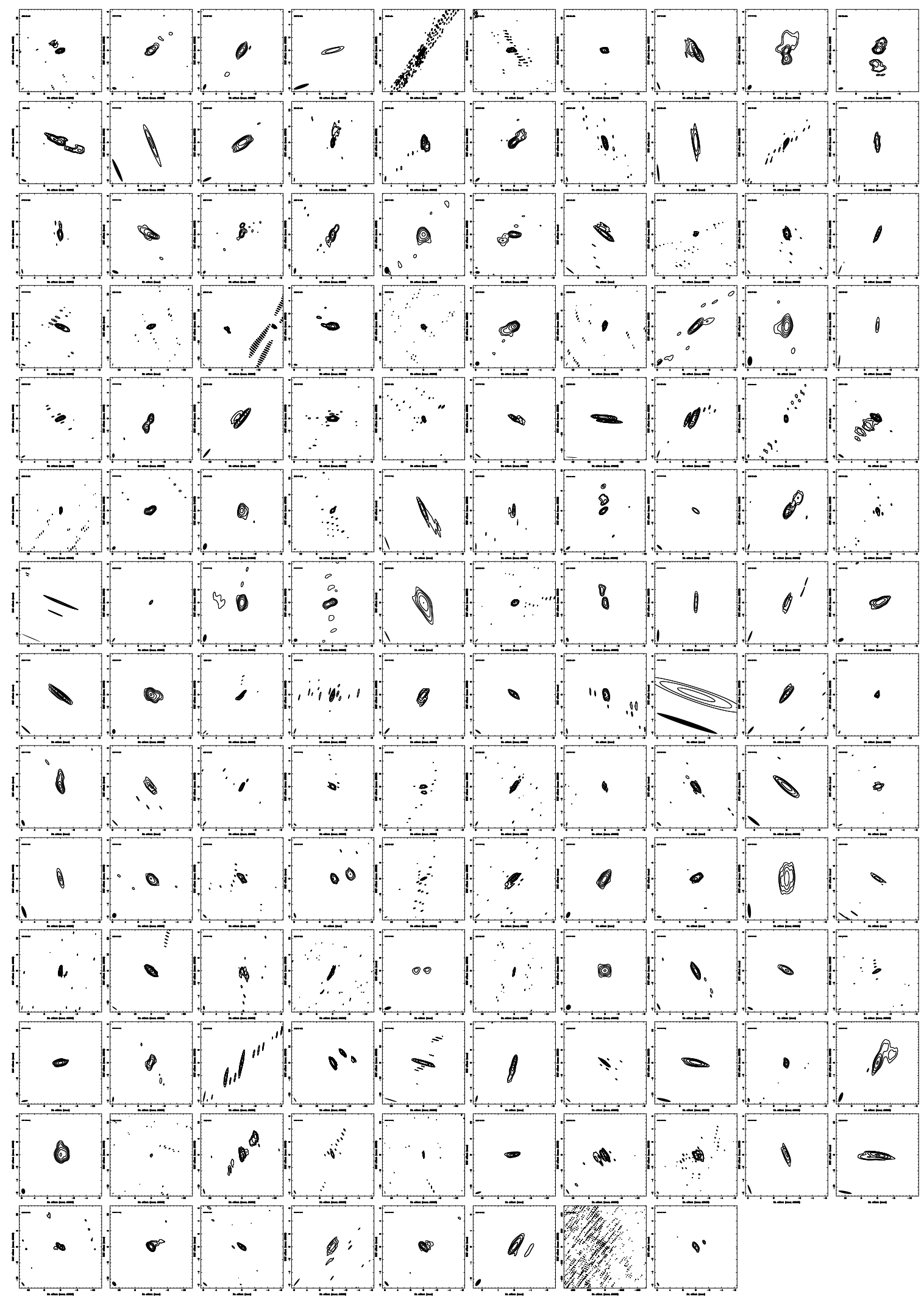

Figure 2: A montage of all the images which will appear in the VSOP Survey Paper V. 